\title{
高能碰撞末态粒子相空间分形特性与HURST 指数的研究
}

\author{
李迪开 ${ }^{(1)}$, 陈刚 ${ }^{(1)}{ }^{*}$, 刘连寿 ${ }^{(2)(3)}$ \\ (1) 中国地质大学数学与物理学院物理系, 武汉 430074; \\ (2) 夸克与轻子物理教育部重点实验室, 华中师范大学, 武汉 430079; \\ (3) 华中师范大学粒子物理研究所, 武汉 430079 \\ *联系人, E-mail: chengang1@ @ cug.edu.cn \\ 收稿日期: 2009-10-07; 接受日期: 2010-01-07 \\ 国家自然科学基金资助项目(批准号: 10775056, 90503001)
}

摘要讨论了高能碰撞末态粒子相空间可能存在双 Hurst 指数或多 Hurst 指数现象问题, 给出了末态粒子三 维相空间分形特性的一般性判据. 用已有的实验数据的结果对其正确性进行了验证. 利用不同的 MC 模拟产 生器产生不同质心能量下的模拟数据, 进行了分析和检验.

关键词高能碰撞, Hurst 指数, 分形特性, 判据, 收缩率

PACS: $13.85 . \mathrm{Hd}, 13.87 . \mathrm{Fh}, 13.66 . \mathrm{Bc}$

高能碰撞多重产生中的大局域起伏现象, 首先 是日美乳胶协作组 ${ }^{[1]}$ 在观测超高能宇宙线中的核作 用时被发现的，继而 NA22 $2^{[2]}$ 等实验组先后观测到了 大快度起伏的 “钉子”事件. 这种大的起伏显然不是 统计起伏所能解释的. 为此人们相信, 这可能意味着 在高能碰撞的时空演化过程中存在着某种非线性的 动力学起伏, 或者说其内禀的几率密度函数具有分 形的特性. 为观察到这种非线性动力学起伏, Bialas 等人 ${ }^{[3,4]}$ 提出引入归一化阶乘矩, 用以消除统计起伏, 描述其动力学起伏, 从而通过观测阶乘矩的标度不 变性来研究几率密度的分形行为. 此后人们一直在 寻找阶乘矩的标度行为. Ochs ${ }^{[5]}$ 提出高能强子-强子 碰撞末态相空间的分形行为应存在于高维相空间中. $\mathrm{Wu}$ 和 $\mathrm{Liu}^{[6]}$ 提出, 高维相空间的分形是自仿射, 而不 是自相似的. 因此, 应各向异性地分割相空间, 以观
察标度行为. 由

$$
C_{q}\left(\delta x_{a}, \delta x_{b}\right)=\lambda_{a}^{\phi} \lambda_{b}^{\phi} C_{q}\left(\lambda_{a} \delta x_{a}, \lambda_{b} \delta x_{b}\right),
$$

其中 $\lambda_{a}, \lambda_{b}$ 分别是 $a, b$ 方向的收缩率, 它们应满足

$$
H_{a b}=\ln \lambda_{a} / \ln \lambda_{b} .
$$

$H_{a b}$ 即为 Hurst 指数 ${ }^{[7]}$, 它反映了高能强子-强子碰撞 末态相空间的分形特性. Hurst 指数可以通过阶乘矩 投影到一维 ${ }^{[8]}$

$$
F_{q}\left(M_{i}\right)=A_{i}-B_{i} M_{i}^{-\gamma_{i}} \quad\left(i=y, p_{t}, \varphi\right),
$$

时的饱和指数 $\gamma_{i}\left(i=y, p_{t}, \phi\right)$ 得到

$$
H_{i j}=\frac{1+\gamma_{j}}{1+\gamma_{i}}=\frac{\ln \lambda_{i}}{\ln \lambda_{j}} .
$$

如果 Hurst 指数 $H_{i j}=1$ 或 $\gamma_{i}=\gamma_{j}$, 则与 $i, j$ 平面对应 的为自相似分形; 否则, 为自仿射分形. 这一理论已 经得到了强子-强子碰撞和正负电子对撞实验数据的

引用格式: 李迪开, 陈刚, 刘连寿. 高能碰撞末态粒子相空间分形特性与 HURST 指数的研究. 中国科学: 物理学 力学 天文学, 2010, 40: $440 \sim 447$ 
证实. 已有的研究结果表明: 强子-强子碰撞末态粒 子相空间是自仿射分形 ${ }^{[0 ~ 12]}$; 在能量等于 $91.2 \mathrm{GeV}$ 时, 正负电子对撞末态粒子相空间是自相似的分形 结构 ${ }^{[13 ~ 15]}$, 而一般能量下, 正负电子对撞末态粒子 相空间也是自相似的分形结构 ${ }^{[16]}$.

值得注意的是：最近，我们用蒙特卡罗产生器 Jetset7.4 产生正负电子对撞事件, 在质心能量从 30 $200 \mathrm{GeV}$ 区域中, 对其末态粒子相空间的非线性起伏 进行了能量依赖性的研究. 其研究结果表明, 通过一 维饱和指数计算得到的不同平面的 Hurst 指数 $H_{i j}$ 并 不等于 1 , 而对末态粒子相空间分别进行各向同性地 分割 $\left(\lambda_{1}=\lambda_{2}=\lambda_{3}\right)$ 或各向异性地分割 $\left(\lambda_{1} \neq \lambda_{2} \neq \lambda_{3}\right)$; 在不同的能量和不同方式的相空间分割下, 三维的 $\mathrm{NFM}$ 与分割数的对数之间都呈很好的线性关系 ${ }^{[17]}$, 即都存在反常标度行为

$$
F_{q}(M) \propto M^{\phi}, \quad M \rightarrow \infty .
$$

这表明, 对于正负电子对撞产生的末态粒子相 空间可能存在有两组不同的 Hurst 指数, 我们称之为 双 Hurst 指数 ${ }^{[17]}$. 那么, 这种双 Hurst 指数现象的存在 是否具有普遍意义? 由于双 Hurst 指数现象的存在, 如何确定高能碰撞中末态粒子系统的分形特性?

本文证明了高能碰撞末态粒子相空间存在双 Hurst 指数或多 Hurst 指数现象, 给出了末态粒子三维 相空间存在分形特性的一般性判据. 用已有的实验 结果以及利用不同的 $\mathrm{MC}$ 模拟产生器产生数据, 对得 到的结论进行了分析和验证.

\section{1 存在多Hurst指数现象的讨论}

Hurst 指数 ${ }^{[7]}$ 是定义在一个平面上的, 因此, 在 高能碰撞末态粒子相空间, 一个 Hurst 指数可以反映 或描述一个平面的标度特性或分形特征. 对于高维 相空间, 例如三维相空间, 它的标度特性或分形特征 需要用三个不同的 Hurst 指数才能完全描述. 即

$$
H_{a b}=\frac{1+\gamma_{b}}{1+\gamma_{a}} ; H_{b c}=\frac{1+\gamma_{c}}{1+\gamma_{b}} ; H_{c a}=\frac{1+\gamma_{a}}{1+\gamma_{c}},
$$

这里 $a, b, c$ 分别代表三个不同的相空间方向. 方程(6) 可以展开表示为如下方程组:

$$
\begin{aligned}
& H_{a b} \gamma_{a}-\gamma_{b}=1-H_{a b}, \\
& H_{b c} \gamma_{b}-\gamma_{c}=1-H_{b c}, \\
& H_{c a} \gamma_{c}-\gamma_{a}=1-H_{c a} .
\end{aligned}
$$

方程(7)为以 $\gamma_{a}, \gamma_{b}, \gamma_{c}$ 为变量的一阶线性方程组. 如果其对应的系数行列式不为零, 即 $H_{c a} H_{a b} H_{b c}$ $-1 \neq 0$, 方程(7)有唯一的非零解, 可解得 $\gamma_{a}=\gamma_{b}=$ $\gamma_{c}=-1$, 此解显然无物理意义. 当 $H_{c a} H_{a b} H_{b c}-1=0$ 时, 则方程(7)可能有无穷多组解, 这意味着任何有 物理意义的饱和指数 $\gamma_{a}, \gamma_{b}, \gamma_{c}$ 都可能满足方程组 (7). 这表明，描述高能碰撞末态粒子三维相空间的 Hurst 指数可能有多组, 而不只一组, 只要满足

$$
H_{\mathrm{ca}} H_{\mathrm{ab}} H_{\mathrm{bc}}=1,
$$

即可.

\section{2 分形特性与Hurst指数}

对于一个具有三维分形标度特性的阶乘矩 (NFM)，方程(1)可以表示为

$$
\begin{aligned}
& F_{q}\left(\lambda_{a} \delta x_{a} \lambda_{b} \delta x_{b}, \lambda_{c} \delta x_{c}\right) \\
& =\lambda_{a}^{-\phi_{q}} \lambda_{b}^{-\phi_{q}} \lambda_{c}^{-\phi_{q}} F_{q}\left(\delta x_{a}, \delta x_{b}, \delta x_{c}\right),
\end{aligned}
$$

即

$$
\begin{aligned}
F_{q}\left(\Delta x_{a}, \Delta x_{b}, \Delta x_{c}\right) & =\left(\lambda_{a} \lambda_{b} \lambda_{c}\right)^{-\phi_{q}} F_{q}\left(\delta x_{a} \delta x_{b}, \delta x_{c}\right) \\
& =(\lambda)^{-\phi_{q}} F_{q}\left(\delta x_{a} \delta x_{b}, \delta x_{c}\right),
\end{aligned}
$$

其中 $\lambda_{a}, \lambda_{b}, \lambda_{c}$ 是分别沿 $a, b, c$ 三个方向的收缩率, $\lambda=\lambda_{a} \lambda_{b} \lambda_{c}$ 为三维相空间的总收缩率. 将方程(4)代入 (10)式，可得

$$
F_{q}\left(\Delta x_{a}, \Delta_{b}, \Delta x_{c}\right)=\left(\lambda_{a}^{1+H_{b a}+H_{c a}}\right)^{-\phi_{q}} F_{q}\left(\delta x_{a} \delta x_{b}, \delta x_{c}\right) .
$$

若三维相空间的动力学起伏是各向同性的, 测 量得到的三个一维阶乘矩的饱和指数必然是相等的 (即 $\gamma_{a}=\gamma_{b}=\gamma_{c}$ ). 根据方程(6), 有 $H_{a b}=H_{b c}=H_{c a}=1$. 由于 Hurst 指数都等于 1 , 当取收缩率 $\lambda_{a}=\lambda_{b}=\lambda_{c}$ 对 相空间进行各向同性分割时, 可以观测到阶乘矩 $(\mathrm{NFM})$ 的标度特性. 与各向同性动力学起伏对应的三 维相空间的总收缩率定义为

$$
\lambda^{i s o}=\lambda_{a} \lambda_{b} \lambda_{c}=\lambda_{a}^{3} .
$$

相反地, 若三维相空间的动力学起伏是各向异 性的, 测量得到的三个一维阶乘矩的饱和指数是不 相等的, 即 $\gamma_{a} \neq \gamma_{b} \neq \gamma_{c}$, 有 $\lambda_{a} \neq \lambda_{b} \neq \lambda_{c}$. 根据方程(6), 有 $H_{a b} \neq H_{b c} \neq H_{c a} \neq 1$. 因此, 当我们把由方程(6)计 算得到的 Hurst 指数代入方程(4)确定收缩率, 对相空 间进行分割时, 则可以观测到 NFM 的标度特性. 我 
们定义三维相空间的总收缩率为

$$
\lambda^{\text {aniso }}=\lambda_{a} \lambda_{b} \lambda_{c}=\lambda_{a}^{1+H_{b a}+H_{c a}} .
$$

值得注意的是, 如果在方程(13)中, Hurst 指数满足

$$
H_{b a}+H_{c a}=2 \text {, }
$$

则可以得到

$$
\lambda^{\text {aniso }}=\lambda_{a}^{1+H_{b a}+H_{c a}}=\lambda^{i s o}=\lambda_{a}^{3} .
$$

很显然, 从方程(15)可以看出, 在 Hurst 指数满 足方程(14)的条件下, 对各向异性动力学起伏的相空 间按收缩率 $\lambda_{a}$ 进行各向同性分割, 与由 Hurst 指数确 定的收缩率 $\left(\lambda_{a}, \lambda_{b}, \lambda_{c}\right)$ 对相空间进行各向异性分割时, 得到的总的收缩率相同. 因此, 在一定的条件下, 对 各向异性动力学起伏的相空间按各向同性进行分割 时, 也可以观测到阶乘矩的标度特性. 我们把这种满 足总的收缩率不变的现象, 称之为收缩率守恒.

上述讨论结果表明, 在研究高能碰撞末态粒子 的动力学起伏特性时, 相空间的分割方式和阶乘矩的 标度特性, 并不能够完全确定多粒子末态的分形特 性, 它们应该由 Hurst 指数确定. 即当 Hurst 指数满足

$$
H_{a b}=H_{b c}=H_{c a}=1
$$

时, 若对末态粒子相空间进行各向同性分割, 能够观 测到 NFM 的标度特性, 则高能碰撞多粒子末态系统 是自相似分形的; 否则, 是自仿射分形的. (16)式即 为末态粒子三维相空间分形特性的一般性判据.

\section{Hurst指数饱和指数与标度特性}

根据分形特性的一般性判据, 如果 Hurst 指数满 足方程(16), 根据方程(6), 可以得到三个一维饱和指 数的关系为

$$
\gamma_{a}=\gamma_{b}=\gamma_{c} .
$$

若对相空间进行各向同性分割时, 观察到阶乘 矩 NFM 的标度特性, 则可以判定高能碰撞末态粒子 系统是自相似分形的. 这里方程(16)和方程(17)是等 价的.

如果 Hurst 指数不满足方程(16), 即 $H_{a b} \neq H_{b c} \neq$ $H_{c a} \neq 1$, 三个一维饱和指数的关系为

$$
\gamma_{a} \neq \gamma_{b} \neq \gamma_{c} .
$$

若按 Hurst 指数对相空间进行各向异性分割时, 观察到了 NFM 的标度特性, 可以判定高能碰撞末态 粒子系统是自仿射分形的; 如果 Hurst 指数满足方程
(14)，虽然末态粒子系统仍然是自仿射分形的，但是 对相空间进行各向同性分割时，却可以观察到 NFM 的标度特性. 那么, 在这种条件下饱和指数需要满足 什么条件呢?

将(6)式代入(14)式, 得

$$
H_{b a}+H_{c a}=\frac{1+\gamma_{a}}{1+\gamma_{b}}+\frac{1+\gamma_{a}}{1+\gamma_{c}}=2,
$$

将(19)式变形, 得

$$
\frac{\gamma_{a}-\gamma_{b}}{1+\gamma_{b}}+\frac{\gamma_{a}-\gamma_{c}}{1+\gamma_{c}}=0 .
$$

由于饱和指数都大于零, 在满足 $\gamma_{a} \neq \gamma_{b} \neq \gamma_{c}$ 的 条件下, 方程(20)的解为

$$
\begin{gathered}
\gamma_{a}=f\left(\gamma_{b}, \gamma_{c}\right)=\frac{\gamma_{b}+\gamma_{c}+2 \gamma_{b} \gamma_{c}}{2+\gamma_{b}+\gamma_{c}} \\
\left(\gamma_{b}<\gamma_{a}<\gamma_{c} \text { 或 } \gamma_{c}<\gamma_{a}<\gamma_{b}\right) .
\end{gathered}
$$

如果高能碰撞末态粒子系统的饱和指数满足方程 (21), 虽然末态粒子系统是自仿射分形的，对相空间 进行各向同性分割时, 却可以观察到 NFM 的标度特 性.这表明：对于自仿射分形的末态粒子系统, 若保 持总的收缩率不变, 分别对相空间进行各向同性和 各向异性分割——对应两种不同的 Hurst 指数, 都可 以观察到 NFM 的标度特性, 这种现象称为双 Hurst 指数现象.

\section{4 结果分析与验证}

以上我们证明了高能碰撞末态粒子相空间可能 存在双 Hurst 指数现象, 给出了末态粒子系统三维空 间的分形特性的一般性判据, 以及末态粒子系统的 Hurst 指数、一维 NFM 的饱和指数、NFM 的标度特 性和分形特性的关系. 以下用已有的实验结果以及 不同的 $\mathrm{MC}$ 模拟产生器产生数据, 进行分析和验证.

\section{1 高能强子-强子碰撞和正负电子对撞实验结果 的验证}

在高能强子-强子 $250 \mathrm{GeV} / \mathrm{c} \pi^{+} \mathrm{p}$ 和 $\mathrm{K}^{+} \mathrm{p}$ 碰撞中, 其一维二阶 NFM 参数值如表 1 所示 ${ }^{[9]}$. 从表中可以 计算出, 三个 Hurst 指数满足方程(8), 因此饱和指数 存在有意义的解. 由于 Hurst 指数不满足方程(16), 即 $H_{y p_{T}} \neq H_{p_{T} \phi} \neq H_{\phi y} \neq 1$. 因此, 其末态粒子系统的 动力学起伏是各向异性的, 对应的分形结构是自仿 
射的; 如果对其相空间按 Hurst 指数进行各向异性分 割, 可以观测到 NFM 的标度特性. 由于 $\gamma_{y} \neq \gamma_{p_{T}} \approx \gamma_{\phi}$, 且它们不满足方程(21), 因此, 如果对其相空间进行 各向同性分割, 我们将无法观测到 NFM 的标度特性. 这里的分析结果与实验结果一致 ${ }^{[9]}$.

在质心能量为 $91.2 \mathrm{GeV}$ 的正负电子对撞中, 其 一维二阶 NFM 参数值如表 2 所示 ${ }^{[18 ~ 23]}$. 从表 2 中可 以计算出, 三个 Hurst 指数满足方程(8), 因此饱和指 数存在有意义的解. 在误差范围内 Hurst 指数满足方 程(16), 即 $H_{y p_{T}}=H_{p_{T} \phi}=H_{\phi y}=1$. 因此, 其末态粒子 系统的动力学起伏是各向同性的, 对应的分形结构 是自相似的; 如果对其相空间按 Hurst 指数进行各向 同性分割, 可以观测到 NFM 的标度特性. 这一结果 与实验结果一致 ${ }^{[18 ~ 23]}$.

\section{2 用MC模型Jetset7.4 和Herwig5.9 产生数据的} 分析

我们利用 MC 模拟产生器 Jetset7.4 和 Herwig5.9
产生正负电子对撞事件, 分析检验末态粒子系统三 维相空间的分形特性的一般性判据, 以及未态粒子 系统的 Hurst 指数、一维 NFM 的饱和指数、NFM 的 标度特性和分形特性的关系. 在从 40 200 GeV 的质 心能量范围内, 每隔 $10 \mathrm{GeV}$ 选取一个实验样本, 每 个质心能量下的实验样本包含 1000000 个对撞事件. 选取 $y, p_{t}, \varphi$ 作为相空间变量, 对应的相空间区域为 $-6 \leq y \leq 6 \mathrm{GeV} ; 0 \leq \varphi \leq 2 \pi$.

首先, 我们按以下方程计算一维二阶 $(q=2)$ 归一 化阶乘矩 $F_{2}$ :

$$
F_{q}(\delta y) \equiv \frac{1}{M} \sum_{m=1}^{M} \frac{\left\langle n_{m}\left(n_{m}-1\right) \cdots\left(n_{m}-q+1\right)\right\rangle}{\left\langle n_{m}\right\rangle^{q}}
$$

然后, 根据投影方程(3)拟合得到饱和指数, 其 数据填入表 3 中.

从表 3 中饱和指数 $\gamma_{y}, \gamma_{p_{T}}, \gamma_{\phi}$ 的数据和图 1 中饱 和指数的分布可以看出: 只有当能量等于 $91.2 \mathrm{GeV}$ 时，在误差范围内，三个饱和指数相等; 在一般

表 $1250 \mathrm{GeV} / \mathrm{c} \pi^{+} \mathrm{p}$ 和 $\mathrm{K}^{+} \mathrm{p}$ 碰撞一维二阶 NFM 参数值(数据来自于 NA22 实验组 ${ }^{[9]}$ )

\begin{tabular}{ccccc}
\hline$\gamma_{y}$ & $\gamma_{p_{T}}$ & $\gamma_{\varphi}$ & $H_{y p_{T}}$ & $H_{p_{T} \varphi}$ \\
\hline $1.14 \pm 0.24$ & $0.021 \pm 0.006$ & $0.014 \pm 0.053$ & $0.48 \pm 0.06$ & $0.99 \pm 0.01$ \\
\hline
\end{tabular}

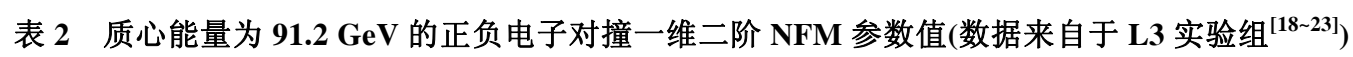

\begin{tabular}{cccccc}
\hline$\gamma_{y}$ & $\gamma_{p_{T}}$ & $\gamma_{\varphi}$ & $H_{y p_{T}}$ & $H_{p_{T} \varphi}$ & $H_{\varphi y}$ \\
\hline $0.966 \pm 0.019 \pm 0.028$ & $0.972 \pm 0.042 \pm 0.030$ & $0.966 \pm 0.034 \pm 0.024$ & $1.003 \pm 0.031 \pm 0.024$ & $0.996 \pm 0.038 \pm 0.027$ & $0.999 \pm 0.027 \pm 0.021$ \\
\hline
\end{tabular}

表 3 正负电子碰撞中不同能量下拟合一维阶乘矩的饱和指数

\begin{tabular}{|c|c|c|c|c|c|c|}
\hline \multirow{2}{*}{$\begin{array}{c}\mathrm{MC} \text { 产生器 } \\
\sqrt{s}(\mathrm{GeV})\end{array}$} & \multicolumn{3}{|c|}{ Jetset7.4 ${ }^{[17]}$} & \multicolumn{3}{|c|}{ Herwig5.9 } \\
\hline & $\gamma_{y}$ & $\gamma_{p_{T}}$ & $\gamma_{\phi}$ & $\gamma_{y}$ & $\gamma_{p_{T}}$ & $\gamma_{\phi}$ \\
\hline 40 & $1.62 \pm 0.02$ & $0.75 \pm 0.04$ & $1.22 \pm 0.07$ & $1.21 \pm 0.04$ & $0.49 \pm 0.05$ & $1.04 \pm 0.08$ \\
\hline 50 & $1.49 \pm 0.02$ & $0.82 \pm 0.04$ & $1.25 \pm 0.05$ & $1.17 \pm 0.04$ & $0.61 \pm 0.06$ & $1.02 \pm 0.07$ \\
\hline 60 & $1.38 \pm 0.02$ & $0.98 \pm 0.04$ & $1.25 \pm 0.04$ & $1.14 \pm 0.03$ & $0.76 \pm 0.06$ & $1.04 \pm 0.05$ \\
\hline 70 & $1.30 \pm 0.01$ & $1.02 \pm 0.05$ & $1.25 \pm 0.03$ & $1.10 \pm 0.02$ & $0.91 \pm 0.06$ & $1.07 \pm 0.04$ \\
\hline 80 & $1.24 \pm 0.01$ & $1.13 \pm 0.05$ & $1.24 \pm 0.03$ & $1.09 \pm 0.02$ & $1.05 \pm 0.09$ & $1.09 \pm 0.04$ \\
\hline 91.2 & $1.17 \pm 0.01$ & $1.17 \pm 0.05$ & $1.19 \pm 0.02$ & $1.05 \pm 0.01$ & $1.21 \pm 0.03$ & $1.04 \pm 0.02$ \\
\hline 100 & $1.14 \pm 0.01$ & $1.29 \pm 0.06$ & $1.19 \pm 0.02$ & $0.99 \pm 0.01$ & $1.32 \pm 0.04$ & $1.10 \pm 0.01$ \\
\hline 110 & $1.11 \pm 0.01$ & $1.34 \pm 0.06$ & $1.17 \pm 0.02$ & $0.83 \pm 0.01$ & $1.45 \pm 0.11$ & $1.14 \pm 0.01$ \\
\hline 120 & $1.07 \pm 0.01$ & $1.34 \pm 0.06$ & $1.15 \pm 0.02$ & $0.75 \pm 0.01$ & $1.57 \pm 0.08$ & $1.13 \pm 0.01$ \\
\hline 130 & $1.05 \pm 0.01$ & $1.38 \pm 0.06$ & $1.12 \pm 0.02$ & $0.71 \pm 0.01$ & $1.52 \pm 0.16$ & $1.10 \pm 0.01$ \\
\hline 140 & $1.02 \pm 0.01$ & $1.44 \pm 0.07$ & $1.11 \pm 0.02$ & $0.68 \pm 0.01$ & $1.58 \pm 0.19$ & $1.07 \pm 0.01$ \\
\hline 150 & $0.998 \pm 0.006$ & $1.42 \pm 0.07$ & $1.12 \pm 0.02$ & $0.65 \pm 0.01$ & $1.50 \pm 0.23$ & $1.05 \pm 0.01$ \\
\hline 160 & $0.974 \pm 0.006$ & $1.42 \pm 0.07$ & $1.11 \pm 0.02$ & $0.63 \pm 0.01$ & $1.66 \pm 0.11$ & $1.02 \pm 0.01$ \\
\hline 170 & $0.955 \pm 0.005$ & $1.54 \pm 0.08$ & $1.10 \pm 0.02$ & $0.61 \pm 0.01$ & $1.59 \pm 0.12$ & $1.00 \pm 0.01$ \\
\hline 180 & $0.940 \pm 0.005$ & $1.53 \pm 0.09$ & $1.09 \pm 0.01$ & $0.60 \pm 0.01$ & $1.58 \pm 0.24$ & $0.98 \pm 0.01$ \\
\hline 190 & $0.915 \pm 0.005$ & $1.52 \pm 0.09$ & $1.08 \pm 0.01$ & $0.59 \pm 0.01$ & $1.68 \pm 0.12$ & $0.96 \pm 0.01$ \\
\hline 200 & $0.902 \pm 0.005$ & $1.53 \pm 0.09$ & $1.07 \pm 0.01$ & $0.59 \pm 0.01$ & $1.16 \pm 0.14$ & $0.94 \pm 0.01$ \\
\hline
\end{tabular}


情况下三个饱和指数都不相等. 这表明, 一般情况下, 正负电子对撞产生的末态粒子系统的动力学起伏是 各向异性的; 只有当能量等于 $91.2 \mathrm{GeV}$ 时, 其末态 粒子的动力学起伏才是各向同性的. 为了验证上述 结果并作进一步的分析, 根据方程(6)计算出三维相 空间的三个不同平面的 Hurst 指数, 填入表 4 中.

从表 4 中的数据可以看出, 当能量等于 $91.2 \mathrm{GeV}$

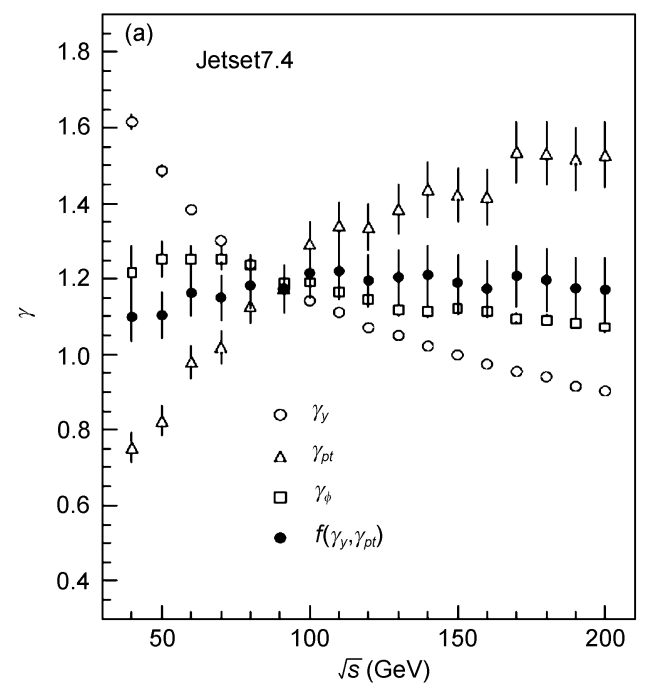

时, 在误差范围内, 三个不同平面的 Hurst 指数相等, 即 $H_{y p_{T}}=H_{p_{T} \phi}=H_{\phi y}=1$, 这意味着末态粒子系统是 自相似的分形结构; 当能量不等于 $91.2 \mathrm{GeV}$ 时, 三 个不同平面的 Hurst 指数并不相同, 即 $H_{y p_{T}} \neq H_{p_{T} \phi}$ $\neq H_{\phi y} \neq 1$ ，这表明，在一般情况下，正负电子对撞末 态粒子系统是自仿射的分形结构.

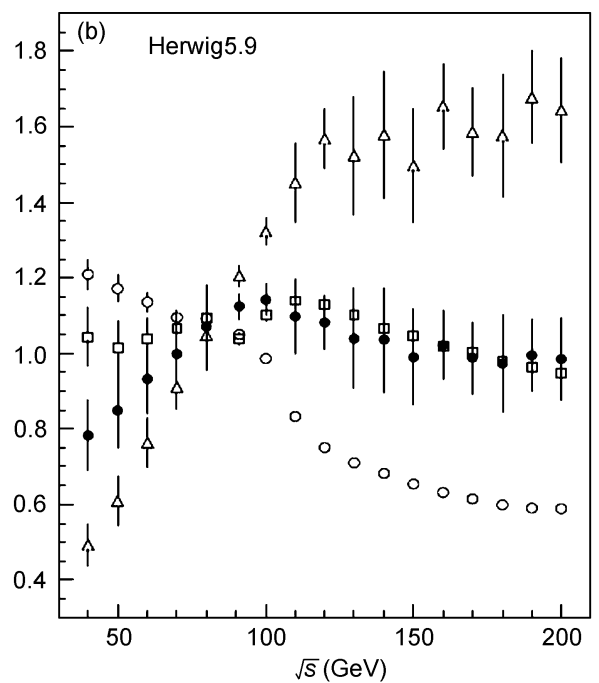

图 1 正负电子碰撞中不同能量下拟合得到的一维阶乘矩的饱和指数的分布图 数据产生(a) Jetset7.4, (b) Herwig5.9, 其中实心点为根据方程(21)计算得到的函数 $f\left(\gamma_{y}, \gamma_{p_{T}}\right)$ 的值

表 4 正负电子碰撞中不同能量下的 Hurst 指数

\begin{tabular}{|c|c|c|c|c|c|c|}
\hline \multirow{2}{*}{$\begin{array}{c}\mathrm{MC} \text { 产生器 } \\
\sqrt{s}(\mathrm{GeV}) \\
\end{array}$} & \multicolumn{3}{|c|}{ Jetset7.4 ${ }^{[17]}$} & \multicolumn{3}{|c|}{ Herwig5.9 } \\
\hline & $H_{\phi y}$ & $H_{\phi p_{T}}$ & $H_{p_{T} y}$ & $H_{\phi y}$ & $H_{\phi p_{T}}$ & $H_{p_{T} y}$ \\
\hline 40 & $1.18 \pm 0.04$ & $0.79 \pm 0.05$ & $1.50 \pm 0.03$ & $1.08 \pm 0.02$ & $0.73 \pm 0.01$ & $1.48 \pm 0.03$ \\
\hline 50 & $1.07 \pm 0.03$ & $0.81 \pm 0.04$ & $1.37 \pm 0.03$ & $1.08 \pm 0.02$ & $0.80 \pm 0.01$ & $1.35 \pm 0.03$ \\
\hline 60 & $1.06 \pm 0.03$ & $0.88 \pm 0.04$ & $1.14 \pm 0.04$ & $1.05 \pm 0.02$ & $0.87 \pm 0.01$ & $1.21 \pm 0.03$ \\
\hline 70 & $1.02 \pm 0.02$ & $0.90 \pm 0.04$ & $1.14 \pm 0.03$ & $1.01 \pm 0.01$ & $0.92 \pm 0.01$ & $1.10 \pm 0.02$ \\
\hline 80 & $1.05 \pm 0.02$ & $0.95 \pm 0.03$ & $1.05 \pm 0.03$ & $1.00 \pm 0.01$ & $0.98 \pm 0.03$ & $1.02 \pm 0.04$ \\
\hline 91.2 & $0.99 \pm 0.02$ & $0.99 \pm 0.04$ & $1.00 \pm 0.03$ & $1.01 \pm 0.01$ & $1.08 \pm 0.01$ & $0.93 \pm 0.01$ \\
\hline 100 & $0.98 \pm 0.01$ & $1.05 \pm 0.04$ & $0.93 \pm 0.03$ & $0.94 \pm 0.01$ & $1.11 \pm 0.01$ & $0.85 \pm 0.01$ \\
\hline 110 & $0.97 \pm 0.01$ & $1.08 \pm 0.04$ & $0.90 \pm 0.02$ & $0.86 \pm 0.01$ & $1.15 \pm 0.04$ & $0.75 \pm 0.03$ \\
\hline 120 & $0.96 \pm 0.01$ & $1.09 \pm 0.04$ & $0.88 \pm 0.03$ & $0.82 \pm 0.01$ & $1.21 \pm 0.03$ & $0.68 \pm 0.02$ \\
\hline 130 & $0.97 \pm 0.01$ & $1.12 \pm 0.04$ & $0.86 \pm 0.03$ & $0.81 \pm 0.01$ & $1.20 \pm 0.07$ & $0.68 \pm 0.04$ \\
\hline 140 & $0.96 \pm 0.01$ & $1.15 \pm 0.04$ & $0.83 \pm 0.03$ & $0.81 \pm 0.01$ & $1.25 \pm 0.09$ & $0.65 \pm 0.05$ \\
\hline 150 & $0.94 \pm 0.01$ & $1.14 \pm 0.04$ & $0.83 \pm 0.03$ & $0.81 \pm 0.01$ & $1.22 \pm 0.11$ & $0.66 \pm 0.06$ \\
\hline 160 & $0.93 \pm 0.01$ & $1.14 \pm 0.04$ & $0.82 \pm 0.03$ & $0.81 \pm 0.01$ & $1.31 \pm 0.05$ & $0.61 \pm 0.02$ \\
\hline 170 & $0.93 \pm 0.01$ & $1.21 \pm 0.05$ & $0.77 \pm 0.03$ & $0.81 \pm 0.01$ & $1.29 \pm 0.05$ & $0.62 \pm 0.03$ \\
\hline 180 & $0.93 \pm 0.01$ & $1.21 \pm 0.05$ & $0.77 \pm 0.04$ & $0.81 \pm 0.01$ & $1.30 \pm 0.12$ & $0.62 \pm 0.06$ \\
\hline 190 & $0.92 \pm 0.01$ & $1.20 \pm 0.05$ & $0.76 \pm 0.04$ & $0.81 \pm 0.01$ & $1.37 \pm 0.06$ & $0.59 \pm 0.03$ \\
\hline 200 & $0.92 \pm 0.01$ & $1.22 \pm 0.05$ & $0.75 \pm 0.04$ & $0.82 \pm 0.01$ & $1.36 \pm 0.06$ & $0.60 \pm 0.03$ \\
\hline
\end{tabular}


为了对以上结论作进一步的检验, 以下研究三 维 NFM 的标度特性. 当能量等于 $91.2 \mathrm{GeV}$ 时, 动力学 起伏是各向同性的, 因此, 如果对末态粒子相空间进 行各向同性分割, 应该观测到三维 NFM 的标度特性. 当能量不等于 $91.2 \mathrm{GeV}$ 时, 动力学起伏是各向异性 的, 因此, 按表 4 中的 Hurst 指数对末态粒子相空间 进行各向异性分割时, 能够观测到三维 NFM 的标度 特性. 根据表 4 中的 Hurst 指数对相空间进行各向异 性分割时, 计算得到质心能量为 $40 \sim 200 \mathrm{GeV}$ 时, 三 维 2 5 阶 NFM, 将其中能量分别为 $80,120,180 \mathrm{GeV}$ 时 NFM 的分布图画在图 2 中.

在图 2 中, 是用 Jetset7.4 和 Herwig5.9 两种不同 的模型产生的不同能量的数据样本计算得到的三维 2 5 阶 NFM 分布. 虽然因非整数分割时丢掉了末尾 的小的相空间, 其分布产生了局部性的起伏 ${ }^{[11,12]}$, 但 $\ln F_{q} \sim \ln M$ 分布仍然呈现出比较好的标度特性. 这 表明, 正负电子碰撞末态粒子系统, 在能量不等于
$91.2 \mathrm{GeV}$ 时, 其分形结构都是自仿射的.

从图 1 中(a)和(b)可以看出, 三个一维饱和指数 满足方程(21), 即在误差范围内满足 $\gamma_{\phi}=f\left(\gamma_{y}, \gamma_{p_{T}}\right)$. 因此, 如果对正负电子对撞末态粒子相空间按表 4 中 的 Hurst 指数进行各向异性分割与对末态粒子相空间 进行各向同性分割时的总的收缩率相等. 所以, 如果 对末态粒子相空间进行各向同性分割时，也应该观 测到三维 NFM 的标度特性.对相空间进行各向同性 分割, 计算得到质心能量为 $40 \sim 200 \mathrm{GeV}$ 时, 三维 2 5 阶 NFM, 将其中能量分别为 $80,120,180 \mathrm{GeV}$ 时 NFM 的分布图画在图 3 中.

从图 3 中可以看出: 用 Jetset7.4 和 Herwig5.9 两 种不同的模型产生的不同能量的数据样本, 并对相 空间进行各向同性分割时, 计算得到的三维 2 5 阶 NFM 分布, 其 $\ln F_{q} \sim \ln M$ 也呈现出很好的标度特性. 这表明, 正负电子碰撞末态粒子系统, 在能量等于 $91.2 \mathrm{GeV}$ 时, 其分形结构是自相似的; 而在一般情况

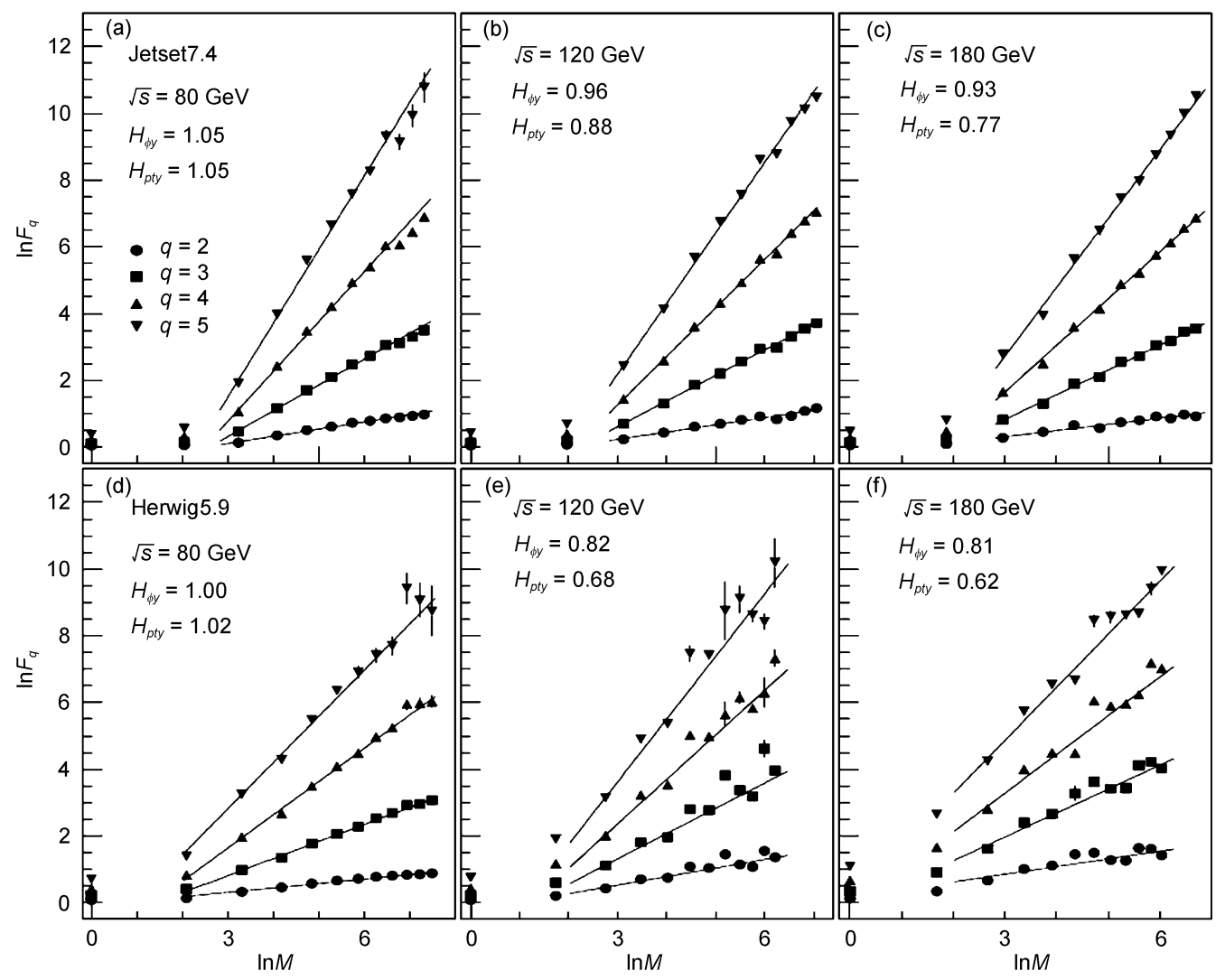

图 2 按 Hurst 指数对相空间进行各向异性分割时的三维 2 5 阶 NFM 分布

其中能量分别为(a)和(d) $80 \mathrm{GeV}$, (b)和(e) $120 \mathrm{GeV}$, (c)和(f) $180 \mathrm{GeV}$; 数据样本分别用 Jetset7.4(a) (c)和 Herwig5.9(d) (f)产生 


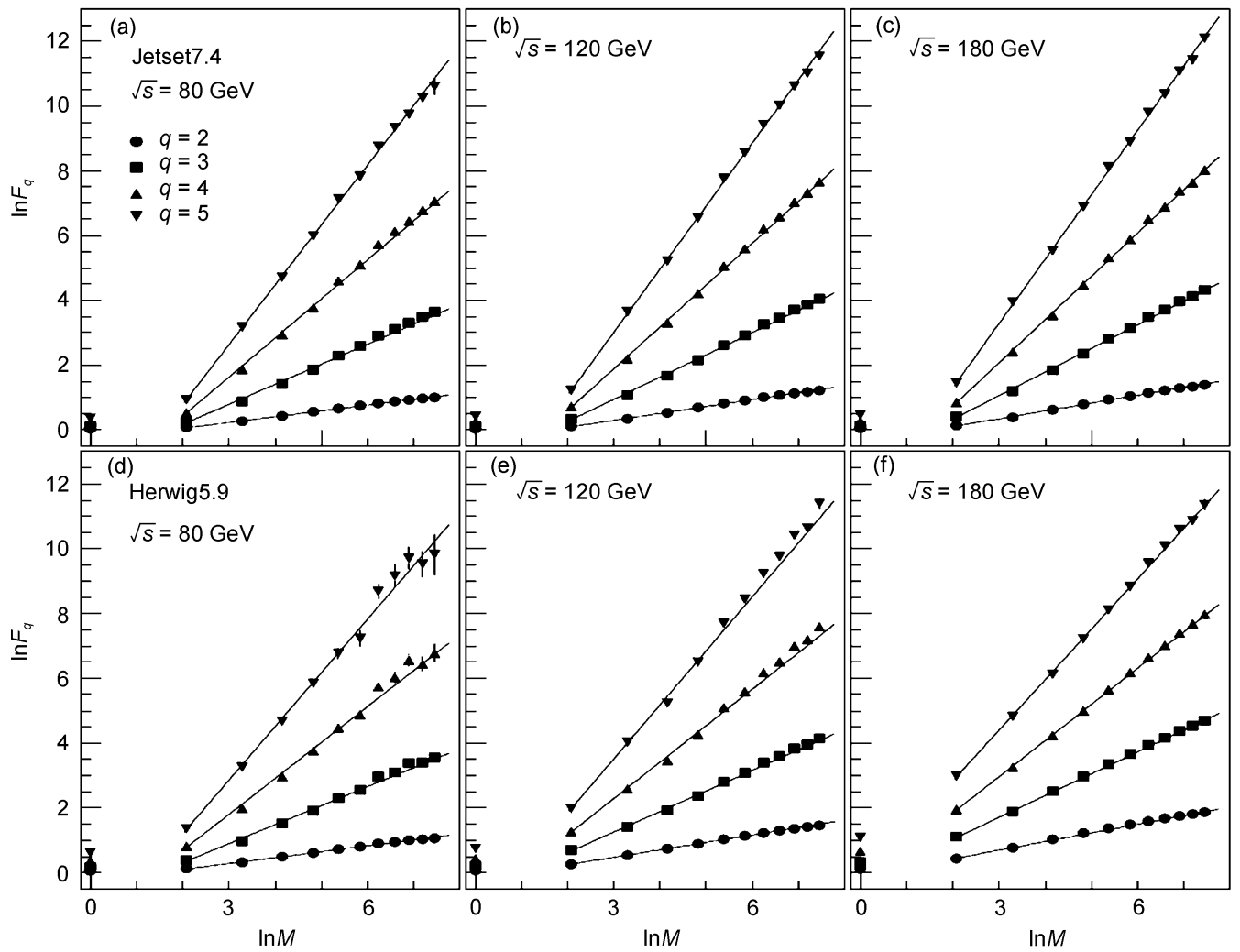

图 3 对相空间进行各向同性分割时的三维 2 5 阶 NFM 分布

其中能量分别为(a)和(d) $80 \mathrm{GeV}$, (b)和(e) $91.2 \mathrm{GeV}$, (c)和(f) $180 \mathrm{GeV}$; 数据样本分别用 Jetset7.4(a) (c)和 Herwig5.9(d) (f)产生

下，虽然其分形结构都是自仿射的，由于按 Hurst 指 数对末态粒子相空间进行各向异性分割与对末态粒 子相空间进行各向同性分割时的总的收缩率相等, 即在不同的能量下总的收缩率是守恒的, 因此, 在各 向同性分割相空间的情况下，仍然能够观测到 NFM 的标度特性.

\section{5 结果与讨论}

本文我们首先讨论了高能碰撞末态粒子相空间 可能存在多 HURST 指数现象, 给出了末态粒子系统 三维相空间的分形特性的一般性判据, 以及末态粒 子系统的 Hurst 指数、一维 NFM 的饱和指数、NFM 的标度特性和分形特性的关系. 并发现, 在一定的条 件下, 只要总的收缩率不变, 对各向异性动力学起伏 的相空间按各向同性进行分割时, 也可以观测到阶 乘矩(NFM)的标度特性. 我们把这种现象称之为收缩 率守恒. 然后, 用已有的实验结果加以验证, 发现原
有实验数据的结果与我们的结论一致. 最后, 用 Jetset7.4 和 Herwig5.9 两种不同的模型, 在不同的质 心能量下模拟产生实验数据, 进行了分析. 发现: 对 于正负电子对撞产生的末态粒子系统, 在能量不等 于 $91.2 \mathrm{GeV}$ 时, 存在双 Hurst 指数现象.

值得注意的是, 正好在质心能量为 $91.2 \mathrm{GeV}$ 时 无双 Hurst 指数现象. 这表明, 当碰撞能量正好在 $Z^{0}$ 能区时, e+e-碰撞部分子级联演化过程是一个自相似 分形过程，而其他能区是自仿射分形过程. 文献[24] 认为 $Z^{0}$ 能区存在自相似分形可能来自于 $Q C D$ 中部分 子簇射的内禀自相似性, 即连续的 $\mathrm{q} \rightarrow \mathrm{qg}$ 和 $\mathrm{g} \rightarrow \mathrm{gg}$ 分 支形成的喷注. 而文献[25]指出在较低能区，2-jet 更多， 这对应于自仿射分形结构, 即纵向的起伏比横向的 起伏更大; 随着能量增加, 3-jet 增多, 2-jet 减少, 即系 统逐步趋向于各向同性; 图 1 中的结果所展示的纵横 方向饱和指数的分布情况与此相符. 在高能区, 随着 质心能量的进一步增加, 2-jet 进一步减少, 而形成第 3 个喷注的胶子越来越硬, 使得横动量及其起伏增加, 
系统在横向的动力学起伏超过纵向, 相空间的演化 又表现出自仿射分形结构. 由于总的收缩率守恒的 限制, 在不同能区形成了双 Hurst 指数现象, 而在能
量为 $91.2 \mathrm{GeV}$ 时, 两套 HURST 指数正好重叠. 是否存 在更本质的动力学因素使得在 $Z^{0}$ 能区两套 HURST 指 数正好重叠, 则有待于作进一步的定量研究和探讨.

\section{参考文献}

1 Burnett T H, Dake S, Fuki M, et al. Extremely high multiplicities in high-energy nucleus-nucleus collisions. Phys Rev Lett, 1983, 50(26): $2062-2065$

2 Adamus M, Ajinenko I V, Amato S F, et al. Maximum particle densities in rapidity space of pi+p, $\mathrm{K}+\mathrm{p}$ and pp collisions at $250 \mathrm{GeV} / \mathrm{c}$. Phys Lett B, 1987, 185(1-2): 200-204

3 Bialas A, Peschanski R. Moments of rapidity distributions as a measure of short-range fluctuations in high-energy collisions. Nucl Phys B, 1986, 273(3-4): 703-718

4 Bialas A, Peschanski R. Intermittency in multiparticle production at high energy. Nucl Phys B, 1988, 308(4): 857-867

5 Ochs W. The importance of phase space dimension in the intermittency analysis of multi hadron production. Phys Lett B, 1990, 247(1): 101-106

6 Wu Y F, Liu L S. Self-affinity of multiplicity fluctuation in the phase space of multiparticle production. Phys Rev Lett, 1993, 70(21): 31973200

7 Mandelbrot B B. Self-affine fractal sets, I: The basic fractal dimensions. In: Family F, Vicsek T, eds. Dynamics of Fractal Surfaces. Singapore: World Scientific Publishing Company, 1991. 21-38

8 吴元芳, 刘连寿. 高能强子-强子碰撞多粒子末态纵向相空间的研究. 中国科学 A 辑: 数学 物理学 天文学, 1994, 37(12): 1299-1307

9 Agababyan N M, Atayan M R, Charlet M, et al. Self-affine fractality in pi+p and K+p collisions at 250 GeV/c. Phys Lett B, 1996, 382(3): 305311

10 Wang S S, Wang Z M, Wu C. Self-affine analysis of multiparticle production in pp collisions at $400 \mathrm{GeV} / \mathrm{c}$ with continuously varying scale. Phys Lett B, 1997, 410(2-4): 323-326

11 Agababyan N M, Atayan M R, Gang C, et al. Self-affine scaling from non-integer phase-space partition in $\pi+p$ and $\mathrm{K}+\mathrm{p}$ collisions at 250 GeV/c. Phys Lett B, 1998, 431(3-4): 451-458

12 Chen G, Liu L S, Gao Y M. On the factorial moment analysis of high energy experimental data with non-integer partition number. Int J Mod Phys A, 1999, 14(23): 3687-3697

13 Abreu P, Adam W, Adye T, et al. Multiplicity fluctuations in hadronic final states from the decay of the Z0. Nucl Phys B, 1992, 386(2): 471—492

14 Akrawy M Z. Intermittency in Hadronic Decays of the Z0. Phys Lett B, 1991, 262(2-3): 351-361

15 Abbiendi G, Ackerstaff K, Alexander G, et al. Intermittency and correlations in hadronic Z(0) decays. Eur Phys J C, 1999, 11(2): 239-250

16 Li D K, Chen G, Wei H L. Energy dependence of non-linear dynamical features in e+e- collisions. Chin Phys C (HEP \& NP), 2008, 32 (7): 552 $-555$

17 Chen G, Li D K, Liu L S. The power spectrum of anomalous scaling in a high-energy e+e- collision and double-Hurst-exponent fractal. J Phys G-Nucl Part Phys, 2008, 35(6): 065002

18 Adeva B, Aguilar-Benitez M, Akbari H, et al. The construction of the L3 experiment. Nucl Instrum Methods Phys Res Sect A, 1990, 289(1-2): $35-102$

19 Bakken J A, Barone L, Blaising J J, et al. High energy cosmic muons and the calibration of the L3 electromagnetic calorimeter. Nucl Instrum Methods Phys Res Sect A, 1989, 275(1): 81-88

20 Adriani O, Arefiev A, An Q, et al. Hadron calorimetry in the L3 detector. Nucl Instrum Methods Phys Res Sect A, 1991, 302(1): 53-62

21 Adeva B, Aguilar-Benitez M, Alcaraz J, et al. A high resolution muon detector. Nucl Instrum Methods Phys Res Sect A, 1992, 323(1-2): 109-124

22 Deiters K, Donat A, Friebel W, et al. The construction and performance of a large cylindrical wire chamber with cathode readout. Nucl Instrum Methods Phys Res Sect A, 1992, 323(1-2): 162-168

23 Acciarri M, Adam A, Adriani O, et al. The L3 silicon microvertex detector. Nucl Instrum Methods Phys Res Sect A, 1994, 351(2-3): 300-312

24 Knowles G, Lafferty G D. Hadronization in Z0 decay. J Phys G-Nucl Part Phys, 1997, 23(7): 731—789

25 JADE Collaboration, Bartel W, Becker L, et al. Experimental studies on multijet production in e+e- annihilation at PETRA energies. Z Phys C-Particles Fields, 1986, 33(1): 12-31 